\title{
Application of Social Support and Social Support Utilization for Chronic Diseases Patients Who Have Depression
}

\author{
Yanfang Deng ${ }^{1}$, Jinai $\mathrm{He}^{2,}$, Yunjia $\mathrm{Li}^{1}$ \\ ${ }^{1}$ Psychiatry Department, The First Affiliated Hospital of Jinan University, Guangzhou, China \\ ${ }^{2}$ Nursing Department, The First Affiliated Hospital of Jinan University, Guangzhou, China \\ Email address: \\ 804434822@qq.com (Yanfang Deng), 1259130133@qq.com (Jinai He), 945063818@qq.com (Yunjia Li) \\ ${ }^{*}$ Corresponding author
}

To cite this article:

Yanfang Deng, Jinai He, Yunjia Li. Application of Social Support and Social Support Utilization for Chronic Diseases Patients Who Have Depression. Science Journal of Clinical Medicine. Vol. 9, No. 2, 2020, pp. 22-25. doi: 10.11648/j.sjcm.20200902.11

Received: April 8, 2020; Accepted: April 24, 2020; Published: May 27, 2020

\begin{abstract}
Objective: To evaluate application of social support and social support utilization for chronic diseases patients who have depression. Methods: 485 patients who were diagnosed as one of 6 chronic diseases, that include diabetes mellitus, stroke, coronary heart disease, malignant tumor, chronic kidney disease or chronic obstructive pulmonary disease were invested to join our study. Their diagnosis of time is from January 2018 to July 2018, and they also suffered from depression during the follow-up period. We used the questionnaires to collect the information from patient, such as patient health questionnaire 9 (C-PHQ-9) which is base on Multidimensional Scale of Perceived Social Support (MSPSS). Result: In all patient characteristics research, the incidence of depressive symptoms was $42.3 \%$, it includes 101 cases of mild depression (49.3\%), 62 cases of moderate depression (30.2\%) and 42 cases of severe depression (20.5\%). Some domains can influence depression in the life, they are education level, course of illness, monthly average medical expenses and self-care ability of patients with chronic depression and non-depression. In addition, some domains are statistical significance in the result, that include total score of social support utilization, perceived friend support, perceived other support, total score of perceived social support, perceived voluntary support, perceived professional support and perceived friendship support. Conclusion: The social support has strongly improvement to depression status of chronic diseases patients. Higher social support utilization improves the patients to better depression situation.
\end{abstract}

Keywords: Social Support, Chronic Diseases, Depression

\section{Introduction}

Depression is a heterogeneous psychiatric disorder that is often as-sociated with other diseases. chronic diseases can contribute to onset of depression as the psychological adjustment to chronic illnesses can be highly challenging [1]. Base on the report, these associations which between chronic diseases and depression appear to be bidirectional: people with depression have an in-creased risk of several chronic conditions, such as diabetes, cardiovascular disease, cancer and stroke [2]. In worldwide, about 350 million people were affected by depression, there had 3\%-16.9\% lifetime prevalence. It accounts for $7.5 \%$ of years lived with disability worldwide, which is the largest contributor to nonfatal health loss [3, 4]. In 2016, the Survey of Mental Disorders in Korea shown the lifetime prevalence of mental disorders disease in South Korea is $5.0 \%$, and the 12 -month prevalence is $1.5 \%$ [5]. In recent report, patients with depression often have comorbid conditions, the comorbid conditions included diabetes, arthritis, asthma, chronic obstructive pulmonary disease, heart disease and hypertension [6]. It is a bidirectional relationship between depression and chronic diseases.

Strong social support, in the form of intimacy, affection, and assistance, has been consistently found to protect against negative mental health outcomes, including depression and anxiety [7]. In social support, it had many channels to communication, such as in-person interactions, such as with family members, significant others and friends. Additionally, social support can also be provided through other communication platforms, such as the Internet and social media, although findings have been mixed with regard to the 
value of such mediated support [8, 9]. Social support is defined as instrumental, informational, affiliative, and emotional support obtained through an individual's social network [10]. A lack of social support is associated with poor health outcomes including increased mental health problems, chronic disease burden and mortality [11]. Aim of this study is that evaluate application of social support and social support utilization for chronic diseases patients who have depression.

\section{Methods}

\subsection{Participants Enrollment and Survey Methods}

485 patients who were diagnosed as one of 6 chronic diseases, that include diabetes mellitus, stroke, coronary heart disease, malignant tumor, chronic kidney disease or chronic obstructive pulmonary disease were invested to join our study. Their diagnosis of time is from January 2018 to July 2018, and they also suffered from depression during the follow-up period. The collected information includes depression status, patient basic information, interpersonal relationship and assessment of social support. In addition, we used the following questionnaires: patient health questionnaire 9 (C-PHQ-9) which is base on The Diagnostic and Statistical Manual of Mental Disorders - IV (DSM-IV), it is similar with Hamilton depression rating scale (HAMD) [12-14]. Additionally, we use the Xiuling Zhou's social support utilization scale to measure the social support utilization of participants [20].

Their inclusion criteria were: (1) the patients were diagnosed as one of 6 chronic diseases; (2) The participant had depression in follow-up; (3) Patients volunteered to participate the study; (4) Primary school education or above. Their withdraw criteria were: (1) Inability to communicate effectively and Severe mental illness; (2) The illness status is too serious.

\subsection{Statistical Analysis}

Our data analyzer performed the statistical analysis by SPSS 22.0. The $\mathrm{P}$ value, t-test and chi-square test were associated with collection result were analyzed. Besides, the mean standard deviation for statistical description.

\section{Result}

In Table 1, it shows the depression situation of patient in the study, there is 205 out of 485 patients with chronic diseases. Overall, the incidence of depressive symptoms was $42.3 \%$, it includes 101 cases of mild depression (49.3\%), 62 cases of moderate depression (30.2\%) and 42 cases of severe depression $(20.5 \%)$.

Table 1. Depression Status of participants.

\begin{tabular}{lll}
\hline Projects & Cases (n) & Percent (\%) \\
\hline Non-depressive mood & 280 & 57.7 \\
Depressed mood & 205 & 42.3 \\
mild & 101 & 20.8 \\
moderate & 62 & 12.8 \\
severe & 42 & 8.7 \\
\hline
\end{tabular}

Base on Table 2, some domains of patient characteristics are statistical significance. Those are education level, course of illness, monthly average medical expenses and self-care ability of patients with chronic depression and non-depression. Other domains are not statistical significance, it shows the patients have similar characteristics on gender, age and income between Non-depressive mood group and Depressed mood group.

Table 2. Patient characteristics in Non-depressive mood group and Depressed mood group.

\begin{tabular}{|c|c|c|c|c|}
\hline Projects & Non-depressive mood group & Depressed mood group & $x^{2}$ & P Value \\
\hline Gender (Male) & 129 & 181 & \multirow{2}{*}{0.697} & \multirow{2}{*}{0.069} \\
\hline Gender (Female) & 76 & 99 & & \\
\hline Age $(<45$ years old $)$ & 25 & 26 & \multirow{4}{*}{7.098} & \multirow{4}{*}{0.069} \\
\hline Age (45 years old) & 56 & 78 & & \\
\hline Age (60 years old) & 83 & 140 & & \\
\hline Age ( $\geq 75$ years old $)$ & 41 & 36 & & \\
\hline Unemployment (n) & 81 & 104 & \multirow{2}{*}{0.596} & \multirow{2}{*}{0.616} \\
\hline No unemployment (n) & 124 & 176 & & \\
\hline Family monthly income $(<2000)$ & 15 & 21 & \multirow{4}{*}{4.988} & \multirow{4}{*}{0.112} \\
\hline Family monthly income (2000 ) & 31 & 30 & & \\
\hline Family monthly income $(5000 \sim)$ & 89 & 108 & & \\
\hline Family monthly income (10000 ) & 70 & 121 & & \\
\hline Completely Self-sufficient & 81 & 215 & \multirow{4}{*}{70.461} & \multirow{4}{*}{0.000} \\
\hline Partial Self-sufficient & 91 & 52 & & \\
\hline In Need of Great Help & 26 & 9 & & \\
\hline Totally Dependent & 7 & 4 & & \\
\hline Average monthly medical expenses $(<2000)$ & 103 & 193 & \multirow{4}{*}{ - } & \multirow{4}{*}{ - } \\
\hline Average monthly medical expenses (2000 ) & 37 & 34 & & \\
\hline Average monthly medical expenses (5000 ) & 28 & 24 & & \\
\hline Average monthly medical expenses (10000 ) & 37 & 29 & & \\
\hline
\end{tabular}


The Table 3 include 10 domains in our research result, they indicate the social support situation of participants and their social support utilization. Overall, a part of domains is statistical significance in the result, that include total score of social support utilization, friend support, other support, total score of perceived social support, obligation support, professional support and friendship support.

Table 3. Social support score in Non-depressive mood group and Depressed mood group.

\begin{tabular}{llllll}
\hline Projects & Family support & Friends support & Other support & Social support & total score of perceived social support \\
\hline Depressed $(\mathrm{n}=205)$ & $5.81 \pm 0.95$ & $4.24 \pm 1.41$ & $4.58 \pm 1.49$ & $4.88 \pm 1.03$ & $1.83 \pm 0.45$ \\
Non-depressive $(\mathrm{n}=280)$ & $5.84 \pm 0.91$ & $4.65 \pm 1.34$ & $4.91 \pm 1.21$ & $5.12 \pm 0.99$ & $1.99 \pm 0.42$ \\
$\mathrm{t}$ & 0.330 & 0.332 & 0.269 & 4.186 & 3.854 \\
P value & 0.742 & 0.001 & 0.007 & 0.000 & 0.00 \\
\hline
\end{tabular}

Table 3. Continued.

\begin{tabular}{|c|c|c|c|c|c|}
\hline Projects & Obligation support & Friendship support & Professional support & Moral support & Social support utilization \\
\hline Depressed $(n=205)$ & $1.62 \pm 0.48$ & $1.31 \pm 0.43$ & $1.77 \pm 0.67$ & $1.67 \pm 0.33$ & $5.81 \pm 0.95$ \\
\hline Non-depressive $(\mathrm{n}=280)$ & $1.75 \pm 0.50$ & $1.41 \pm 0.48$ & $1.88 \pm 0.64$ & $1.79 \pm 0.34$ & $5.84 \pm 0.91$ \\
\hline $\mathrm{t}$ & 2.736 & 2.477 & 1.773 & 2.594 & 0.330 \\
\hline$P$ value & 0.006 & 0.014 & 0.077 & 0.010 & 0.742 \\
\hline
\end{tabular}

\section{Discussion}

Social support is generally defined as the provision of aid by various social network members. A large body of literature suggests higher level of social support may mitigate or "buffer" the maladaptive behavioral outcomes among individuals exposed to stress, such as alcohol use dis-order and substance abuse [15]. In recent report, social support buffering against negative mental or behavioral health outcomes has examined the construct from different aspects, including objective support, subjective support and source of support [16]. A report shown that greater subjective support buffers the negative health outcomes among individuals exposed to traumatic events or greater psychosocial stressors [17]. However, Carnford's report indicate that objective support seems to have no buffering effect or even have negative effect on mental health [18]. In addition, although objective social support may enhance the coping effect through provision of practical resources, this type of support can also create indebtedness and guilt which deteriorates the psychosocial distress of the support receivers and therefore reduces its potential of stress buffering [19].

Base on above research result, the social support has strongly improvement to depression status of chronic diseases patients. Higher social support utilization improves the patients to better depression situation. In particular, some domains can efficiency improve the mental health of patient in social support, it includes total score of social support utilization, friend support, other support, total score of perceived social support, obligation support, professional support and friendship support. Additionally, some factors of life exacerbate depression, such as education level, course of illness, monthly average medical expenses and self-care ability. In limitation, the study has other influence factors which can affect the result of research, but we have not plan to analyze the factors and exclude them. Additionally, the depression situation of participant lacks more detailed assessment, we only distinguish them in 3 level from general identification.

\section{Funding}

The research study was financially supported by the funding as awarded by the Guangdong Nursing Association (Item no.: gdhl xueh2019zx 303).

\section{References}

[1] Mcelroy E, Napoleone E, Wolpert M, Patalay P. E Clinical Medicine structure and connectivity of depressive symptom networks corresponding to early treatment response. EClinical Medicine. 2019; 8: 29-36.

[2] Borsboom D, Cramer AOJ. Network analysis: an integrative approach to the structure of psychopathology. Annu. Rev. Clin. Psychol. 2017; 9 (1): 91-121.

[3] WHO, Depression and Other Common Mental Disorders: Global Health Estimates. 2017.

[4] Rush AJ, Rush AJ. The varied clinical presentations of major depressive disorder. 2007; 9086: 4-10.

[5] Ministry of Health and Welfare, The Survey of Mental Disorders in Korea. 2016.

[6] Boing F, Melo GR, Boing AC, Moretti-Pires RO, Peres KG, Peres MA. Association between depression and chronic diseases: results from a population-based study, Rev. Saude Publica. 2018; 46: 617-623.

[7] Dougall AL, Hyman KB, Hayward MC, Mc Feeley S, Baum A. Optimism and Traumatic Stress: The Importance of Social Support and Coping 1. Journal of Applied Social Psychology. 2016; 31 (2): 223-245.

[8] Trepte S, Dienlin T, Reinecke L. Influence of social support received in online and offline contexts on satisfaction with social support and satisfaction with life: A longitudinal study. Media Psychology. 2015; 18 (1): 74-105. 
[9] Hale CJ, Hannum JW, Espelage DL. Social support and physical health: The importance of belonging. Journal of American College Health. 2015; 53 (6): 276-284.

[10] Barker, G. Adolescents, Social Support and Help-Seeking Behaviour Consultation with Recommendations for Action. World Heal. Organ. 2007: 1-64.

[11] Hernandez D, Reitzel L, Wetter D, McNeill L. Social support and cardiovascular risk factors among black adults. Ethn. Dis. 2016; 24: 444-450.

[12] Spitzer RL, Kroenke K, Williams JB. Validation and utility of a self-report version of PRIMEMD: the PHQ primary care study. Primary Care Evaluation of Mental Disorders. Patient Health Questionnaire. Jama 1999; 282 (18): 1737-1744.

[13] Hamilton M. Development of a rating scale for primary depressive illness. Br. J. Soc. Clin. Psychol. 1967; 6: 278-296.

[14] Zimet GD, Dahlem NW, Zimet SG, Farley GK. The Multidimensional Scale of Perceived Social Support. Journal of Personality Assessment. 1988; 52: 30-34.

[15] Tang CS-k, Wong WC, Leung PM, Chen W-q, Lee A, Ling DC. Health compromising behaviors among Chinese adolescents: role of physical abuse, school experience, and social support. J. Health Psychol. 2018; 16 (3): 457-466.
[16] Dai W, Chen L, Tan H, Wang J, Lai Z, Kaminga AC, Liu A. Association between social support and recovery from post-traumatic stress disorder after flood: a13-14 year follow-up study in Hunan, China. BMC Publ. Health. 2016; 16 (1): 194 .

[17] Mitchell I, Evans L, Rees, T., Hardy L. Stressors, social support, and tests of the buffering hypothesis: effects on psychological responses of injured athletes. Br. J. Health Psychol. 2019; 19 (3): 486-508.

[18] Cranford JA. Stress-buffering or stress-exacerbation? Social support and social undermining as moderators of the relationship between perceived stress and depressive symptoms among married people. Pers. Relat. 2016; 11 (1): 23-40.

[19] Gleason ME, Iida M, Shrout PE, Bolger N. Receiving support as a mixed blessing: evidence for dual effects of support on psychological outcomes. J. Pers. Soc. Psychol. 2018; 94 (5): 824.

[20] Xiuling Z. Self-help organization participation and social support utilization of malignant tumor patients. Shanghai normal university. 2016. 